\title{
Spectral Analysis of Output Voltage for Buck and Boost DC-DC Converter
}

\author{
Zoran Zlatev and Nikolay Hinov \\ Department of Power Electronics, Faculty of Electronic Engineering and Technologies \\ Technical University of Sofia \\ 8 Kliment Ohridski blvd., 1000 Sofia, Bulgaria \\ hinov@tu-sofia.bg
}

\begin{abstract}
When improving the stability of the electronic devices that we use every day, we often have to make some analysis in order to see the behavior of the electronic device under certain work conditions. Although often the output voltage is at first look in range that seems to be regular, when we test the device, it is of great importance to make specific analysis such as "Spectral Analysis" for closer look at the highest peaks in specific time intervals. There are several software platforms that provide such type of analysis. In our case "LabView" by National Instruments will be used.
\end{abstract}

Key words: Frequency, amplitude, power spectrum, FFT.

\section{INTRODUCTION}

One of the most commonly used converter in electronics is the DC-DC converter. The main characteristic of this converter is that the energy flows from power supply to the load with a closed key and that is why it is called "straight converter".

Figure 1 presents the scheme of a Buck DC-DC converter. In this particular case we can see that a MOS transistor is used as a key. Also this model includes a transistor $\mathrm{T}$ as a switch which will be controlled, a valve - diode D that is uncontrolled, and a filter capacitator $\mathrm{C}$ and a filter inductance L.

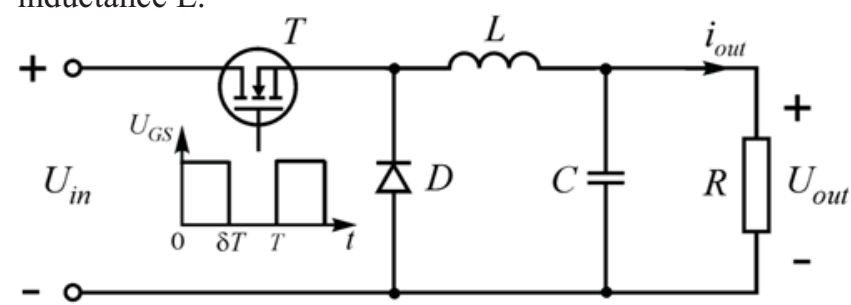

Fig.1 Buck DC-DC Converter

Figure $2 \mathrm{a}$ and $2 \mathrm{~b}$ present equivalent circuits of a straight down converter, a) in the case of an turn-on transistor; b) with a turn-off transistor.

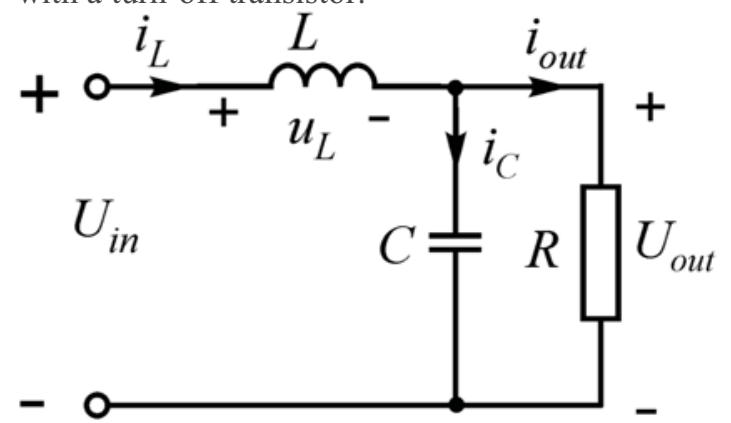

Fig.2a. Case with turn-on transistor

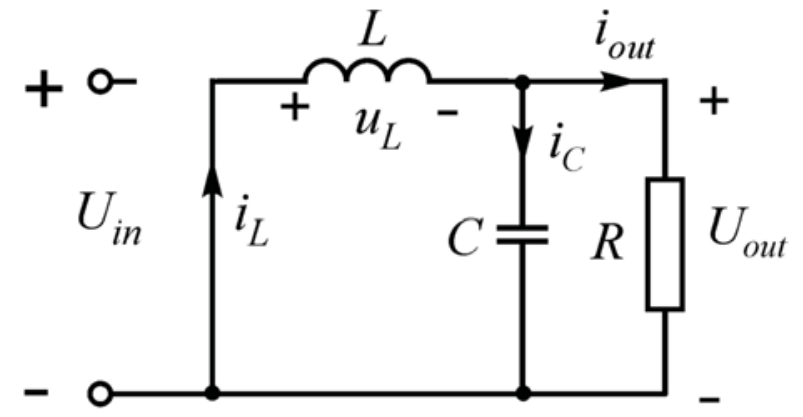

Fig. 2b. Case with turn-off transistor

The $\mathrm{T}$ transistor is controlled with rectangular impulses with frequency $f$ and duty cycle $\mathrm{D}$. When the transistor is open, there is working flow through the coil L to the load. At that time, energy is collected in the coil. With the increase of energy flow in the coil, voltage is determined $U_{\text {in }}-U_{\text {out }}$ which is applied on the coil, inductivity state L and the time while the transistor is switched on. When the transistor is switched off, the energy that is collected in coil L is directed to the load and the $\mathrm{D}$ diode is turned of. There follows a time slot through which current flows to the output of the converter (load and filter capacitor $\mathrm{C}$ ).

The second DC-DC converter that we want to mention in this study is the Boost DC-DC converter. It is called "inverse rising transducer". The use of this DC-DC converter is also very important and can be found in the electric vehicles industry. Most often it is used in regenerative braking of the DC motors in electric cars.

The working scheme of the converter in Figure 4, in an established mode, consists of two basic states of the circuit: one with a drained transistor, the other with a blocked transistor, as shown in Figures $5 \mathrm{a}$ and $5 \mathrm{~b}$.

At the study the values of the elements of which the circuits are comprised, are not taken into account at this point (transistor, diode, capacitor and coil), and also the frequency dependence of their parameters. On the other hand, their reading in most of the cases does not complicate further while modeling $[1,2]$

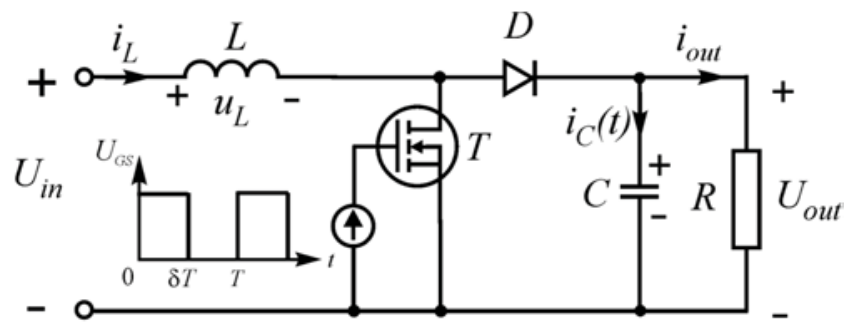

Fig.4. Boost DC-DC converter 


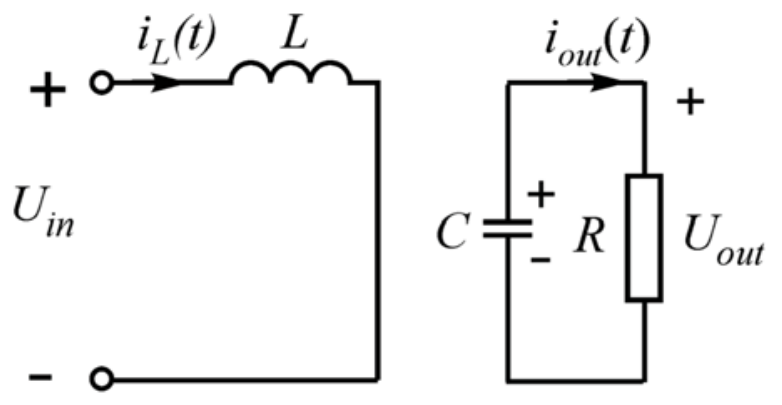

Fig.5a. State with turn-on transistor

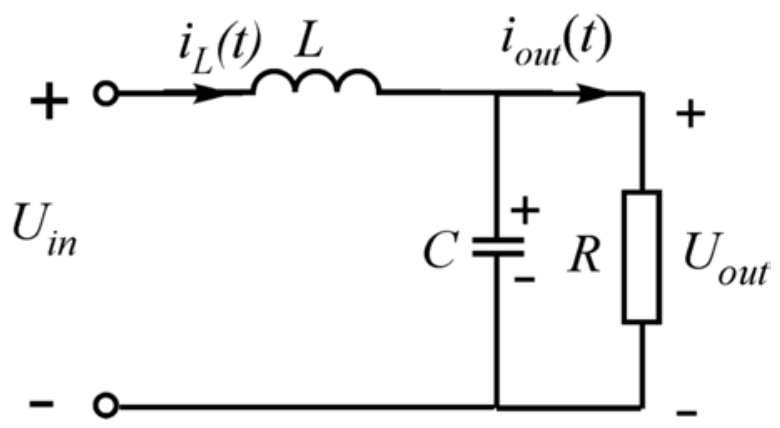

Fig.5b. State with turn-off transistor

In situations when the transistor is drained through the coil, a linear increasing current flows. The increase of this current is determined by the supply voltage $U_{\text {in }}$ which is applied on the coil, the state of the inductivity L, and the time during which the transistor is open. The current flowing through the coil determines the energy accumulated in it. When $\mathrm{T}$ is turned on, the energy that was collected, is going to the load through the diode $\mathrm{D}$. The flow through the coil decreases linearly.

\section{LABVIEW APPROACH}

The following figures present Buck and Boost DC-DC converters models, created in LabView. Figures 7, 8 and 9 describe the design and working of the buck DC-DC converter and also power spectrum analysis of the results that are taken as example at the moment. Figures 10, 11, and 12 describe the working of the boost DC-DC converter, the model scheme and also the power spectrum of the output voltage. These are the results that are taken as an example.

The character of the results is not the main aspect of the study. There are a lot of preferences for adjusting the results for the power spectrum analysis, the future users that do research in the field of electronics will use this filter and power spectrum analysis as needed.

It is important to mention that LabVIEW provides a lot of filter and signal processing functions for the needs of the researchers. FFT, Butterworth, Chebyshev, InverseFFT and a lot other functions for better and faster understanding of the circuits that are analyzed. [2, 3, 4]

Figures 9 and 12 show the power spectrum of the sin wave of the output voltage that we are studying. When scientists want to see how energy is distributed across the frequency, one of the best tool to do that is LabView. The power spectrum shows the energy distribution of a certain magnitude depending on the frequency.

Figures 13 and 14 describe the FFT function of the output voltage of Buck and Boost DC-DC converter. The Fast-
Fourier Transform is one of the most important functions when one describes a signal. Fast - Fourier Transform builds a relationship between the time - domain functions and frequency - domain functions. [4, 5] Inudctor Current

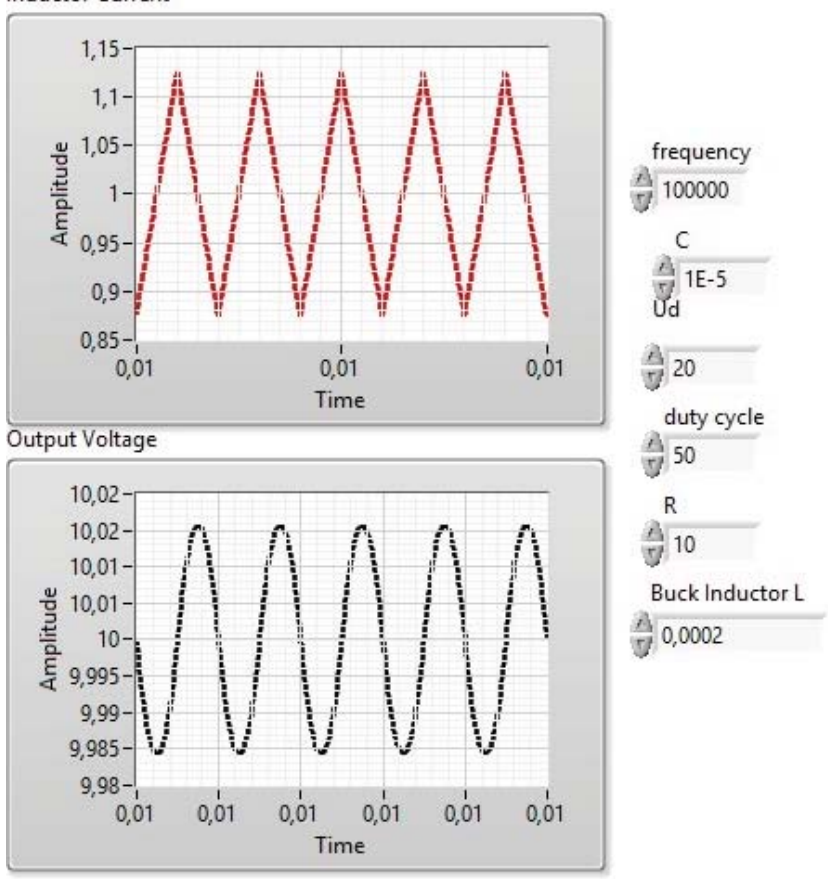

Fig.7. Waveform graph for Buck DC-DC converter

Figures 15 and 16 show the FFT (RMS) or Fast - Fourier Transform of the Root Mean Squared, which is a way of presenting the average mean power of the signal. The RMS value is of great importance because it is the main parameter describing the strength of the alternating current. It is also a measure of energy content in a certain signal.

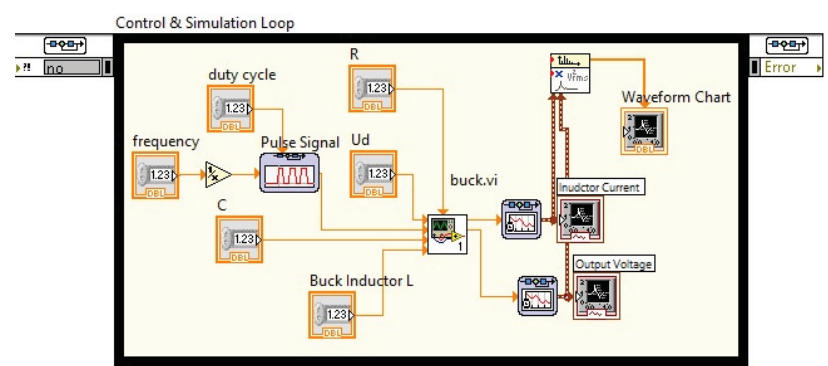

Fig.8. Object - oriented model scheme for Buck DC-DC converter

Figures 17 and 18 describe the phase spectrum. This is also an interesting parameter to examine because the phase is one of the most common parameters when we use Fourier Transform to gather some characteristics of a signal. If we analyze the slope of phase response, we could make predictions of the relative stability, which is very useful when one designs a system.

Figure 19 describes the model scheme of the spectral measurements. This model scheme is a completely different VI from the VI where we present the signals (Figure 8), which is also one of the main functionalities of LabVIEW. With the model scheme in Figure 19 we can import any type of signal and make spectral measurements for the same. These features are useful for making an assessment of the spectrum of the generated signals, which is important to 
obtain electromagnetic compatibility data for the devices even from the level of design and modeling.

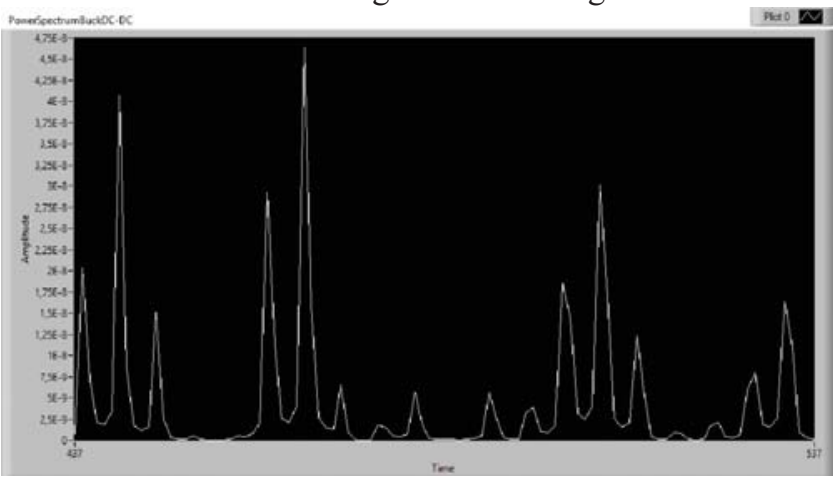

Fig.9. Waveform graph for power spectrum for Buck DCDC converter

In order to see the behavior of the DC converters, the most effective method is to see their spectrograms and power spectrum. They are main tools used to see the qualitative evaluation for the nonlinearity in the results and to better understand these type of converters. The analysis shows the power vs. frequency in diagrams 13 and 14. Inudctor Current

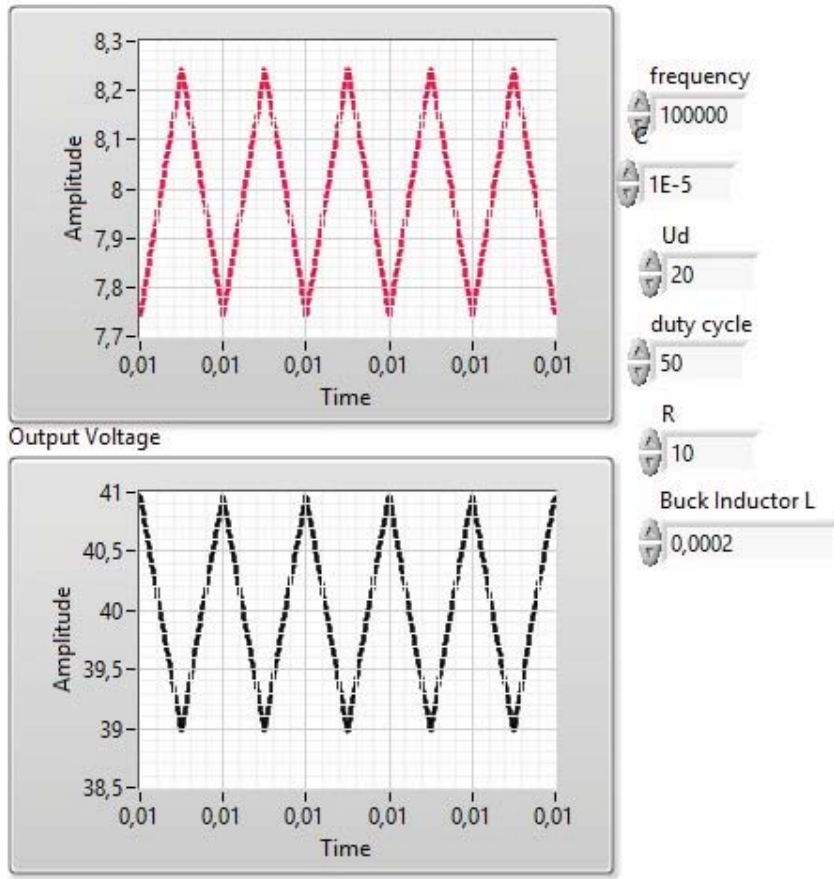

Fig. 10. Waveform graphs for Boost DC-DC converter

Figures 7 and 10 shows the time series with periodic solution.

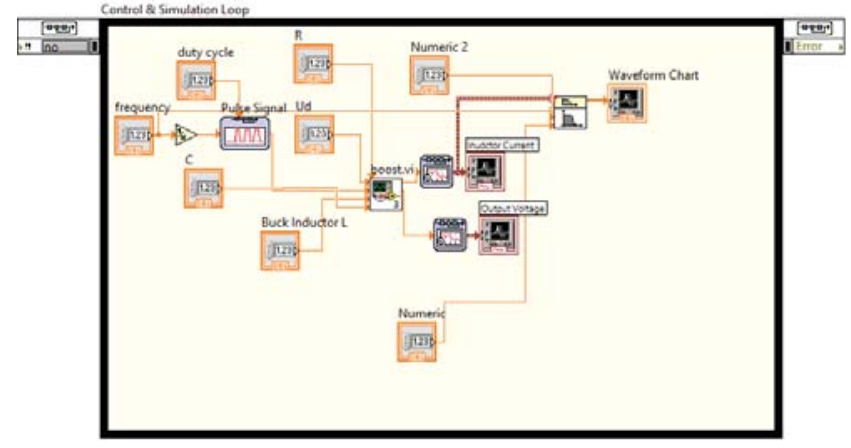

Fig.11. Object - oriented model scheme for Boost DC-DC converter
From the comparison of the results in Figure 9 and Figure 12 , it was found out that at equal frequencies of operation equal values of duty cycle (D), a different spectrum of the output voltage for the two types of converters is obtained.

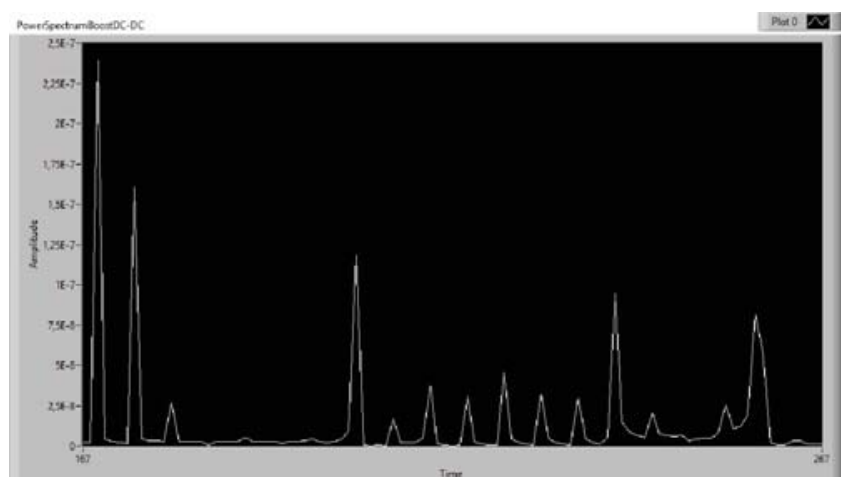

Fig.12. Waveform graph for power spectrum for Boost DCDC converter

Similar results are obtained by comparing the data in Fig. 13 and Fig. 14 about the operation of the two types of power electronic devices. It can be seen that the amplitude of the main harmonic (frequency 0) for the two circuits differs significantly - one is 100 and the other 1600 . This is mainly due to the different principle of operation of the power circuits - one (Buck) is smoothed out by an LC filter, and the other (Boost) inductance $\mathrm{L}$ has the role to increase the input voltage, and the smoothing of the harmonics is done only by the C-filter capacitor.

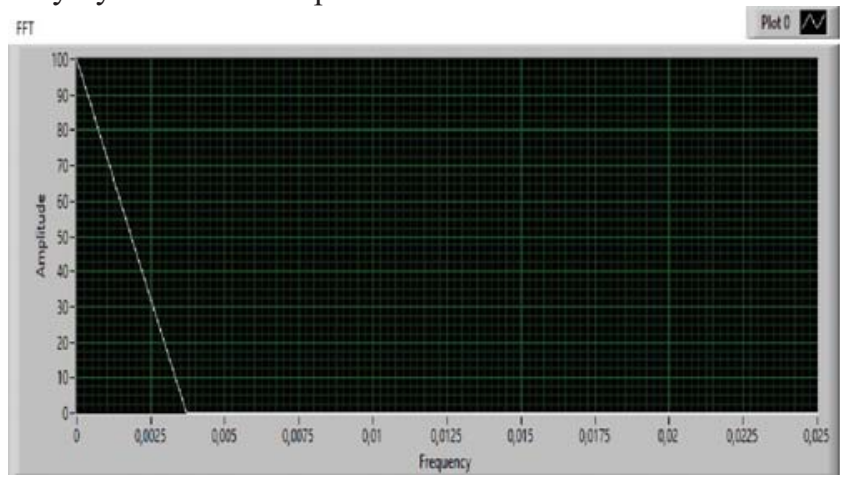

Fig.13. FFT spectrum for Buck DC-DC converter

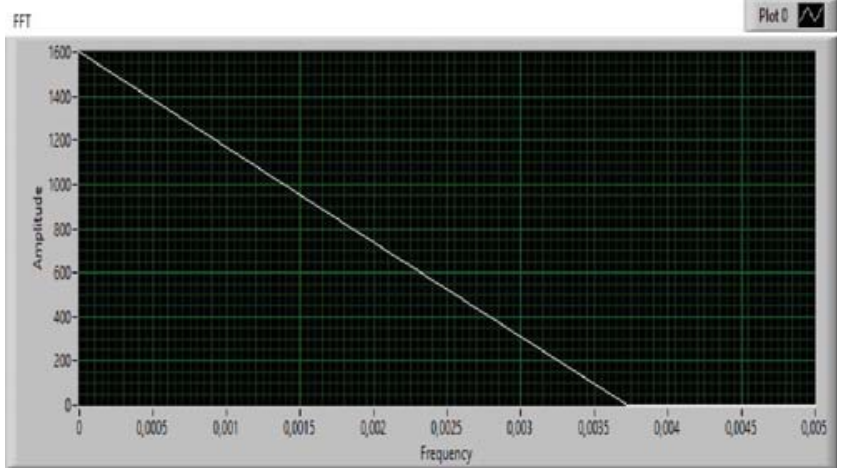

Fig.14. FFT spectrum for Boost DC-DC converter

These results are interesting in order to obtain optimal values of the circuit elements, the main aim being to guarantee the outputs of the converters and also to achieve good dynamics - the possibility to reach in the established mode of operation for the shortest possible time. In this way, the synthesis of the regulator is also essential, which is very important for the safe operation of the devices. 


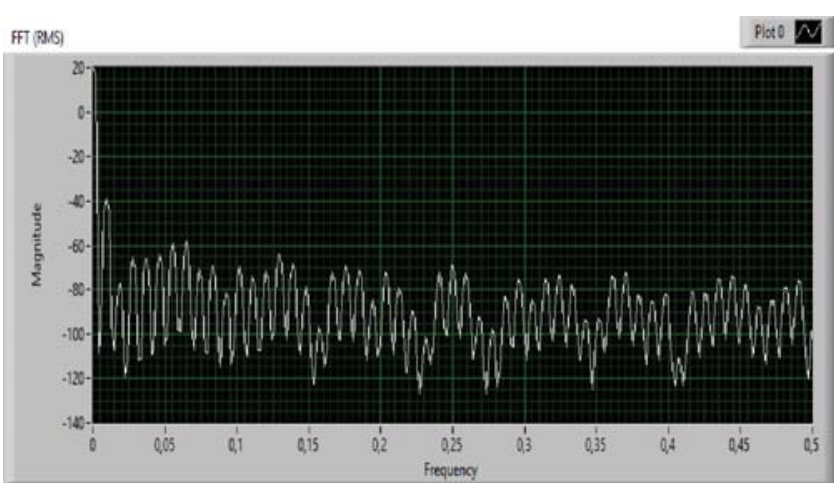

Fig.15. FFT(RMS) spectrum for Buck DC-DC converter

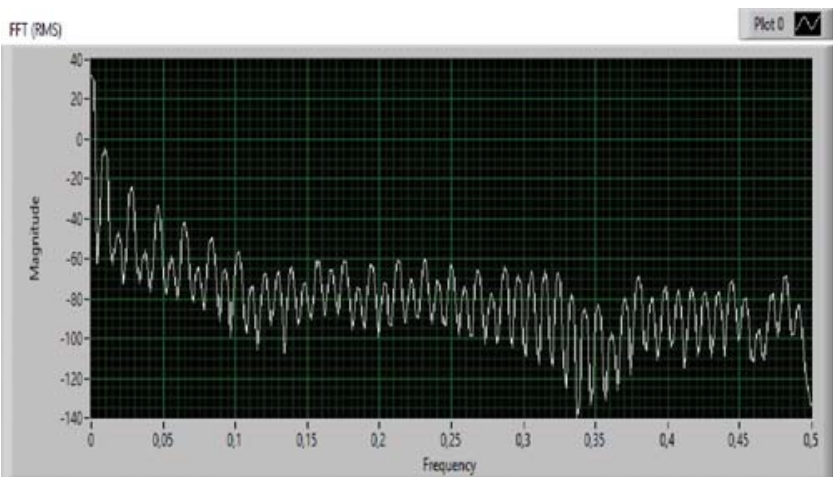

Fig.16. FFT(RMS) spectrum for Boost DC-DC converter

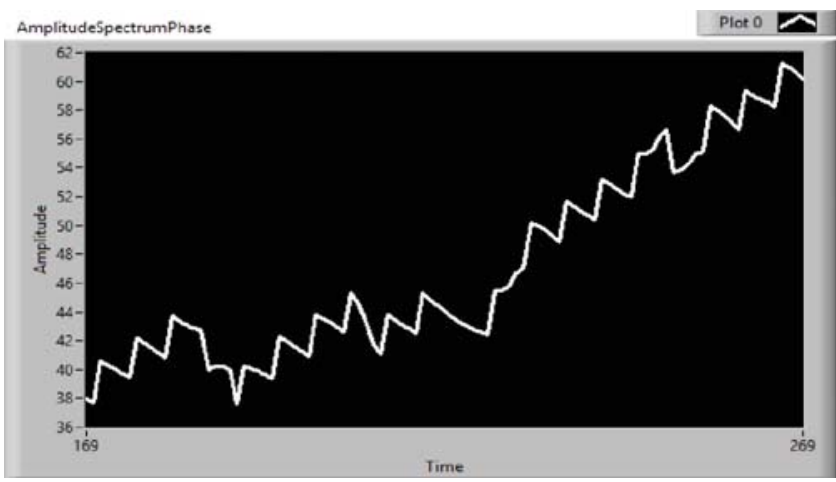

Fig.17. Amplitude Phase Spectrum for Buck DC-DC converter

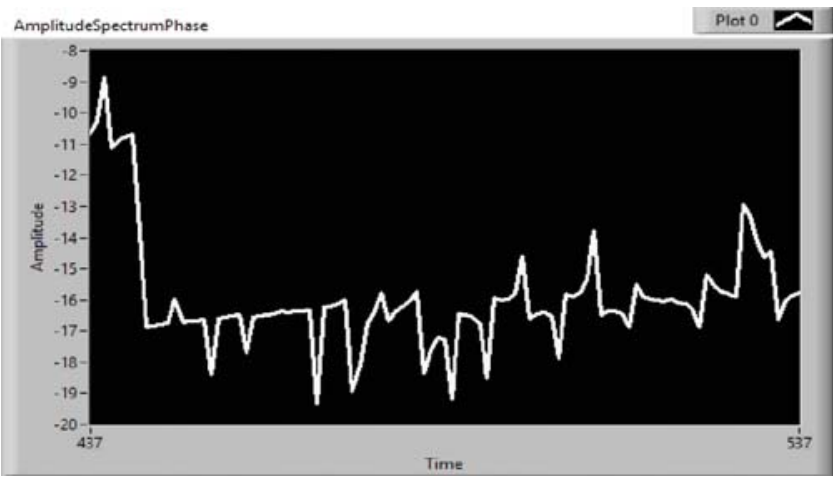

Fig.18. Amplitude Phase Spectrum for Boost DC-DC converter

When examining the phase characteristics of the converters (Figure 17 and Figure 18), there is also a lack of similar results. While in the first scheme the phase is always positive the increase in the second scheme it is negative.

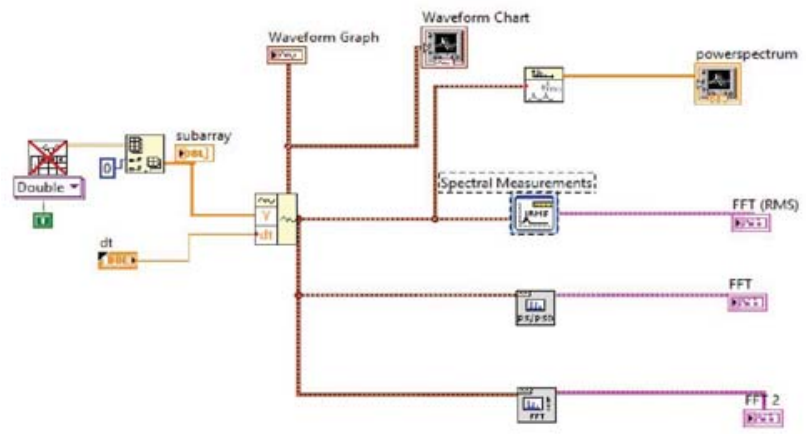

Fig.19. Object - Oriented model scheme design for spectral measurements of the output voltage

\section{CONCLUSION}

The digitalized era of living and exponential growth in the electronic industry force the scientist to make faster analysis and better predictions. Also, at this moment, the necessity of mixing techniques and different types of data processing is more and more usable and important. Information technologies are increasingly used in the electronic industry. LabVIEW is a type of software that makes both sides of the study possible - the electronic aspect and the object oriented programing techniques. With certain knowledge of these technologies, one can make very accurate analyses by decreasing the time and improving the accuracy. When we produce a signal, or use a signal that is produced by a certain DC-DC converter using the functions from spectral measurements that are in LabView, we can get a lot more information about the signal and its behavior. With every change of the main parameters in Figure 7 and 10, the signal will change and we will be able to see how other parameters will change and depending on the results obtained it is very easy to choose parameters that provide for the signal behavior desired by the scientists.

\section{ACKNOWLEDGEMENT}

The research has been carried out within the framework of project "Model based design of power electronic devices with guaranteed parameters", ДН07/06/15.12.2016, of the Bulgarian National Scientific Fund.

\section{REFERENCES}

[1] A. F. Harvey and Michael Cerna, "The Fundamentals of FFT-Based Signal Analysis and Measurement in LabVIEW and LabWindows", (National Instruments, Application Note 041

[2] A. V. Oppenheim and G. C. Verghese, "Introduction to Communication, Control, and Signal Processing" spring 2010

[3] F.W.Isen, Synthesis Lectures on Signal Processing, 2008, ISBN: 9781598298918

[4] H. Asadi and J. Hudgins, Computer Techniques for Dynamic Modeling of DC-DC Power Converters, 2018, ISBN:9781681734187.

[5] J. Zhao, Automating Measurement Bench of DC-DC Converter in LabVIEW, Electric power and Automation engineering, Shanghai University of electric power 2103, Pingliang-Road, Yangpu, Shanghai, 200090, China

[6] National Semiconductor Application Note 255, "Power Spectra Estimation", November, 1980

[7] S. V. Vaseghi, "Advanced Digital Signal Processing and Noise Reduction, Second Edition”, 2000.

[8] www.ni.com. 